\title{
Serum pyridoxal in patients with rheumatoid arthritis
}

\author{
C. R. SANDERSON, R. E. DAVIS, AND C. E. BAYLISS \\ From the Royal Perth Hospital, Perth, Western Australia
}

\begin{abstract}
Sanderson, C. R., Davis, R. E., and Bayliss, C. E. (1976). Annals of the Rheumatic Diseases, 35, 177-180. Serum pyridoxal in patients with rheumatoid arthritis. Abnormalities of tryptophan metabolism have been reported in patients with rheumatoid arthritis (RA) and it has been suggested that these abnormalities are the result of disordered vitamin B6 metabolism. Fasting serum pyridoxal, assayed by an automated microbiological system, was found to be below normal in 35 out of 42 patients with RA while a similar abnormality was found in 8 out of 35 patients with osteoarthrosis (OA). Within the RA group the abnormality could not be related to the age, sex, or drug therapy of individuals but of the 8 patients with $\mathrm{OA}$ and a low serum pyridoxal, 7 were receiving indomethacin either alone or in conjunction with aspirin.
\end{abstract}

Abnormalities of tryptophan metabolism have been reported in patients with rheumatoid arthritis (RA) (McMillan, 1960; Bett, 1962a; and Pinals, 1964), and Bett (1962b) has further shown that pyridoxine in large doses produced a reduction in these abnormalities in the majority of patients. McKusick and others (1964) reported decreased pyridoxine excretion in RA patients with objective clinical improvement after therapy with pyridoxine hydrochloride.

Many drugs used in the management of rheumatic diseases have the reputation of being ulcerogenic and we have recently reported (Sanderson and Davis, 1975) low serum pyridoxal in patients with active gastric ulceration compared to normal levels in duodenal ulceration. We therefore felt it would be of interest to investigate serum pyridoxal levels in patients with arthritis, in particular comparing rheumatoid with osteoarthrosis (OA), and secondly, to assess the effect of drug therapy of these conditions on serum pyridoxal.

\section{Materials and methods}

Blood was taken from fasting patients and the serum separated and stored at $-20^{\circ} \mathrm{C}$ until vitamin levels were assayed. Serum pyridoxal was assayed by an automated microbiological technique using Lactobacillus casei as a test organism (Davis, Smith, and Curnow, 1973). Serum B12 was measured by the method of Nicholas and Pitney (1958), and serum folate by the method of Davis, Nicol, and Kelly (1970). Patients with RA fulfilled the diagnostic criteria of the American Rheumatism Association (Ropes and others, 1959) and age, sex, duration of disease, drug therapy, and evidence of previous peptic ulceration was noted on the protocol. The laboratory was unaware of the clinical diagnosis at the time of vitamin assay.

\section{Results}

Seventy-seven patients (18 males, 59 females) were investigated; their ages ranged from 15-77 years, mean 60.5 years. Forty-two had RA and 35 OA. Twelve of the rheumatoid group had negative serum tests for rheumatoid factor. The mean ages of the RA and OA groups were $56 \cdot 3$ and 65.8 years, respectively.

No patient had active peptic ulcer disease at the time of this study although 10 had been previously investigated for dyspepsia. Hiatus hernia had been shown by radiology in 5 , and in 2 of these endoscopy showed no evidence of active peptic ulceration. One patient had had a vagotomy and pyloroplasty performed 3 years previously and another surgery after a perforated duodenal ulcer in 1968 . One patient had a gastric ulcer shown radiologically in 1970, and the remaining 2 patients had normal barium meals within 2 years of this study.

Serum folate (reference range $2 \cdot 5-18 \cdot 3 \mu \mathrm{g} / \mathrm{l}$ ) was below normal in 9 patients and serum pyridoxal below normal in 44. Correlation between these two vitamin levels in individual patients is shown in Fig. 1. 


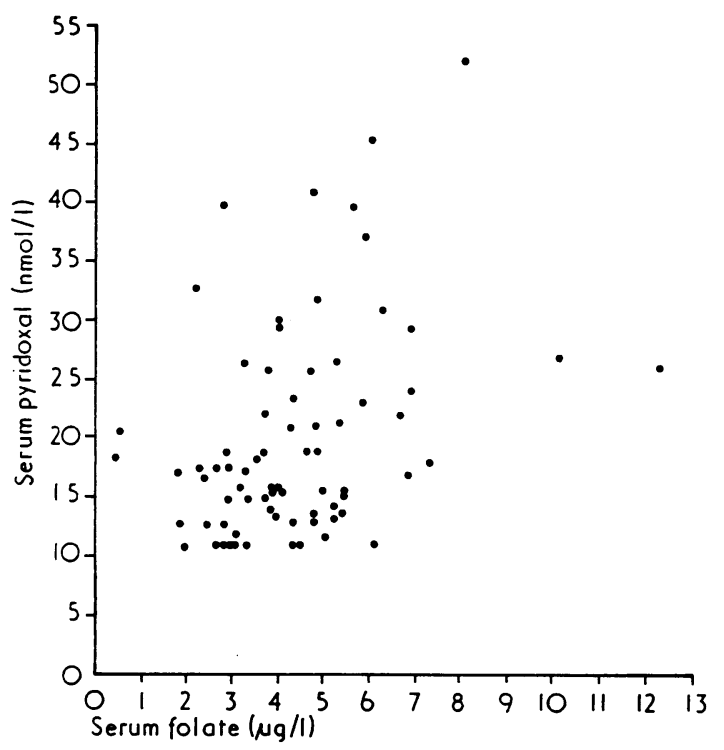

FIG. 1 Serum pyridoxal/serum folate levels in individual patients

Serum B12 was normal in all patients. Serum pyridoxal normally decreases with age and is slightly lower in females. Correlation between age and serum pyridoxal is shown in Figs. 2 (males) and 3 (females).

Serum pyridoxal was below normal in 36 of the 42 patients with RA and in 8 of the 35 patients with OA. Individual values are shown in Fig. 4. Drug therapy varied between one and five different drugs per patient in RA, and none to three in the OA group. Correlation between the number of drugs received per patient and the mean serum pyridoxal in both groups is shown in the Table.

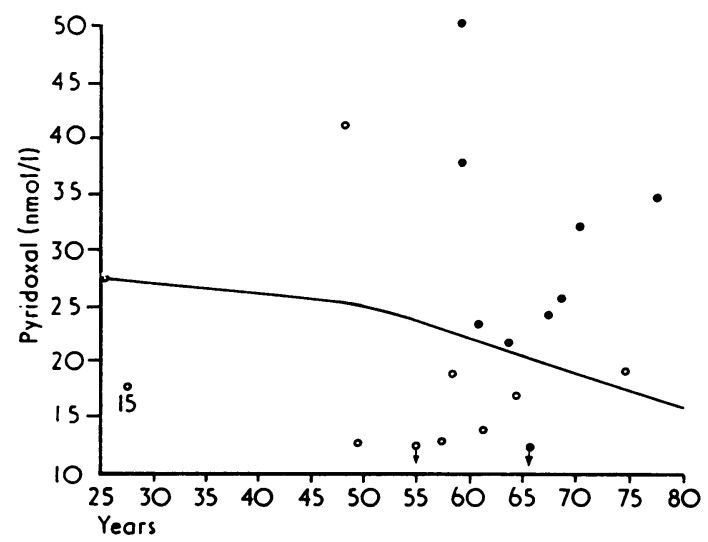

FIG. 2 Serumpyridoxal lage in males. $(O=R A, \bullet=O A)$. The lower limit of the normal range is shown as a continuous line

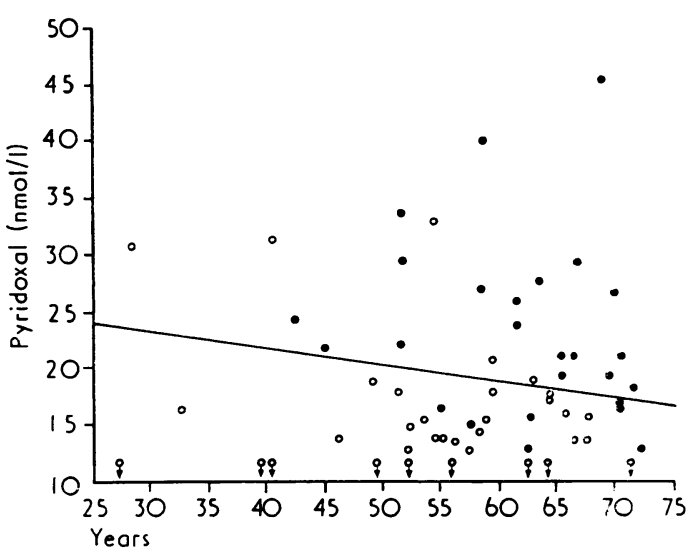

FIG. 3 Serum pyridoxallage in females. $(\circlearrowleft=R A$, $\bullet=O A)$. The lower limit of the normal range is shown as a continuous line

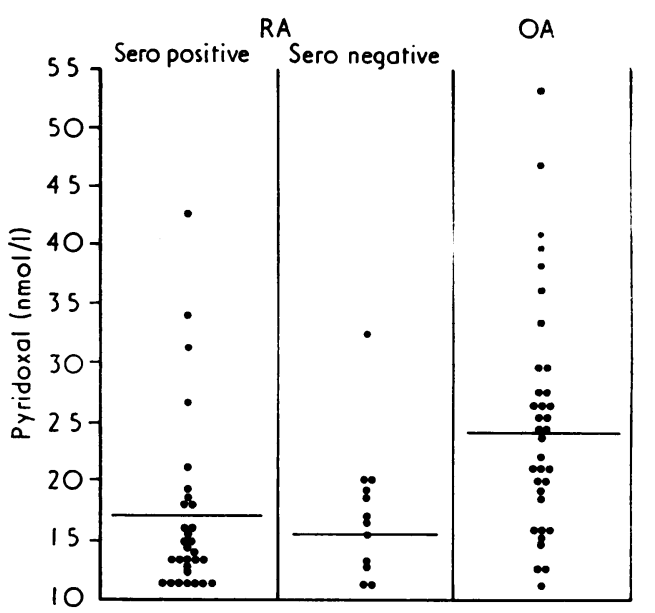

FIG. 4 Serum pyridoxal in individual patients. The mean values for each group are also shown

\section{Discussion}

A low serum pyridoxal level may result from an inadequate diet but no dietary difference was apparent between the RA and OA groups as all were inpatients at the time of this study. However, no detailed dietary studies were performed and perhaps the RA group ate less and/or more selectively during the investigation. Alternatively the low levels may reflect poor nutrition before admission to hospital.

Serum folate was lower in the RA group to a highly significant degree $(P<0.001)$, which could also favour a dietary aetiology. However, serum pyridoxal was below normal in 36 of 42 patients with RA whereas folate was below normal in only 8 ; furthermore no apparent correlation existed between the two vitamin levels in individual patients. In those 8 
Table Correlation between number of drugs taken and mean serum pyridoxal in $R A$ and $O A$ patients

\begin{tabular}{|c|c|c|c|c|c|c|c|}
\hline No. of drugs per patient & 0 & 1 & 2 & 3 & 4 & 5 & Total \\
\hline \multicolumn{8}{|l|}{ RA } \\
\hline No. of patients & - & 7 & 21 & 11 & 2 & 1 & 42 \\
\hline Mean serum pyridoxal* & - & $15 \cdot 7$ & $17 \cdot 3$ & $16 \cdot 3$ & $18 \cdot 7$ & 11 & $16 \cdot 6$ \\
\hline \multicolumn{8}{|l|}{ OA } \\
\hline No. of patients & 6 & 17 & 9 & 3 & 一 & - & 35 \\
\hline Mean serum pyridoxal* & $25 \cdot 4$ & $26 \cdot 2$ & $22 \cdot 7$ & $24 \cdot 0$ & - & - & $24 \cdot 9$ \\
\hline
\end{tabular}

* Because levels $<12 \mathrm{nmol} / \mathrm{l}$ cannot be accurately assayed, values below this figure are assumed to be $11 \mathrm{nmol} / 1$ for the purpose of comparison.

patients with low serum folate the values varied between 0.3 and $2.5 \mu \mathrm{g} / 1$, and serum pyridoxal varied between $<12 \mathrm{nmol} / 1$ and $33 \mathrm{nmol} / 1$; in patients with a serum pyridoxal below $12 \mathrm{nmol} / \mathrm{l}$, serum folate varied from 1.9 to $40 \mu \mathrm{g} / 1$. Three patients who were receiving folate supplements had folate levels $>40 \mu \mathrm{g} / 1$, and serum pyridoxal levels of $<12,31 \cdot 2$, and $29.4 \mathrm{nmol} / \mathrm{l}$. Although no dietary studies were done on these patients, McKusick and others (1964) reported that when standard diets were fed to RA patients and controls, the former excreted significantly less pyridoxine in their urine, suggesting that dietary insufficiency is not the major cause of the low serum pyridoxal found in RA patients.

Serum pyridoxal normally decreases with age but as the mean age of the OA group is $\mathbf{9 . 5}$ years greater than that of the rheumatoid group, this only serves to emphasize the difference in the vitamin levels between the two groups. Females predominate in both groups but males follow the group pattern, and although they have a higher mean value, this is also true of normal population studies.

The role of drug therapy is difficult to analyse; however, no relation appears to exist between the number of drugs being used and the serum pyridoxal level. Only 4 patients in the RA group were on drugs known to interfere with vitamin B6 metabolism: 3 were receiving phenytoin (Hagberg, Hamfelt, and Hansson, 1966) and one D-penicillamine (Hollister and others, 1966). Ibuprofen was used by 25 patients and corticosteroids by 13 in the RA group, compared to 3 and none, respectively, of the OA patients, and these were the only drugs which showed a marked group difference. Within the RA group no difference of serum pyridoxal levels could be found between those patients receiving or not receiving these particular drugs. Eight patients with OA had a low serum pyridoxal and of these, 7 were receiving indomethacin and 3 aspirin preparations. Indomethacin was being used by 14 OA patients; one patient had started this drug 2 days and another 2 weeks before investigation and yet another was receiving this drug as a suppository. In all 3 the serum pyridoxal was normal. Of 7 patients receiving indomethacin alone serum pyridoxal was low in 3 and was normal in 4 patients receiving aspirin alone.

These results suggest that indomethacin therapy may play a role in the aetiology of a low serum pyridoxal level. Lanza, Roger, and Nelson (1975) assessed gastric mucosal lesions in volunteers taking aspirin, indomethacin, ibuprofen, and phenylbutazone and concluded that indomethacin and aspirin induced the most marked reaction. We have recently found (Sanderson and Davis, 1976) that serum pyridoxal is low in patients with gastric pathology, including gastritis, and it may be that the gastric mucosal lesion induced by indomethacin is responsible for the low serum pyridoxal in patients receiving this drug.

These results confirm that vitamin B6 metabolism is abnormal in patients with RA in that the fasting serum pyridoxal is below normal in $86 \%$ of patients. Drugs, with the possible exception of indomethacin, age, and sex did not appear to be of aetiological significance. Dietary factors may be important, but as previous studies suggest that RA patients can adequately absorb vitamin B6 and do not excrete excessive amounts of it, the problem is likely to be one of increased utilization of the active principal pyridoxal-5-phosphate in the disease process. An alternative explanation is that some form of gastric pathology occurs in RA as part of the disease complex itself and that this is the reason for abnormalities of vitamin B6 metabolism. Many RA patients have a history of dyspepsia and peptic ulceration and complications of ulceration are not infrequent. This is generally believed to be closely associated with the drug therapy of arthritis, but it may be that these agents merely augment changes already present.

We should like to acknowledge the assistance of Dr. Phyllis Goatcher, Senior Physician, Rheumatology Department, Royal Perth Hospital, in allowing us to investigate patients under her care, and also that of Mr. John Moulton of the Vitamin Assay Laboratory, and Miss Christine Coleman in the preparation of this paper. 


\section{References}

BETT, I. M. (1962a) Ann. rheum. Dis., 21, 63 (Metabolism of tryptophan in rheumatoid arthritis) (1962b) Ibid., 21, 388 (Effect of pyridoxine on tryptophan metabolism in rheumatoid arthritis)

Davis, R. E., Nicol, D. J., AND Kelly, A. (1970) J. clin. Path., 23, 47 (An automated method for the measurement of folate activity)

_-, Smith, B. K., AND CURNow, D. H. (1973) Ibid., 26, 871 (An automated method for microbiological assay of serum pyridoxal)

Hagberg, B., Hamfelt, A., and Hansson, D. (1966) Acta paediat. scand., 55, 371 (Tryptophan level tests and pyridoxal-5-phosphate levels in epileptic children)

Hollister, L. E., MoOre, F. F., Forrest, F., AND BenNett, J. L. (1966) Amer. J. clin. Nutr., 19, 307 (Anti-pyridoxine effect of D-Penicillamine in schizophrenic men)

Lanza, F., Roger, G., AND Nelson, R. (1975) Gastrointestinal Endoscopy, 21, 103 (An endoscopic evaluation of the effects of non-steroidal anti-inflammatory drugs on the gastric mucosa)

McKusick, A. E., Sherwin, R. W., Jones, L. G., AND Hsu, J. M. (1964) Arthr. and Rheum., 7, 636 (Urinary excretion of pyridoxine and 4-pyridoxic acid in rheumatoid arthritis)

McMillan, M. (1960) J. clin. Path., 13, 140 (The identification of a fluorescent reducing substance in the urine of patients with rheumatoid arthritis. The excretion of 3-hydroxyanthranilic acid in this and other conditions)

Nicholas, D., and Pitney, W. R. (1958) Aust. J. exp. Biol. med. Sci., 36, 603 (Microbiological assay of Vitamin B.12 content of serum using Euglena Gracilis)

Pinals, R. S. (1964) Arthr. and Rheum., 7, 662 (Tryptophan metabolism in rheumatic disease)

Ropes, M. W., Bennett, G. A., CobB, S., Jacox, R., And Jessar, R. A. (1959) Ann. rheum. Dis., 18, 49 (Revision of diagnostic criteria for rheumatoid arthritis)

Sanderson, C. R., AND Davis, R. E. (1975) Gut, 16, 177 (Serum pyridoxal in active peptic ulceration)

- - (1976) Gut (in press) (Serum pyridoxal in patients with gastric pathology) 